Pacific Journal of Mathematics

MAXIMAL IDEALS IN ALGEBRAS OF TOPOLOGICAL 


\title{
MAXIMAL IDEALS IN ALGEBRAS OF TOPOLOGICAL ALGEBRA VALUED FUNCTIONS
}

\author{
WILLIAM J. HERY
}

For a completely regular space $T$, topological algegbra $A$ and algebra $X$, both commutative and having identity, let $C(T, A)=\{f: T \rightarrow A: f$ is continous $\}, C^{*}(T, A)=\{f \in C(T, A): f(T)$ is relatively compact $\}$ and $\mathscr{M}(X)$ be the set of all maximal ideals of codimension one in $X$ endowed with the Gelfand topology (i.e., the weak topology generated by $\{\hat{x}: x \in X\}$, where $\hat{x}(m)=x+m)$. When $A$ is the real numbers, the spaces $\mathscr{M}(C(T, A))(=v T)$ and $\mathscr{M}\left(C^{*}(T, A)\right)(=\beta T)$ are well known. If $A$ is any topological algebra, $t \in T$ and $m \in \mathscr{M}(A)$, then $M_{t, m}=\{f \in C(T, A): f(t) \in m\} \in \mathscr{M}(C(T, A))$, and $(t, m) \rightarrow$ $M_{t, m}$ is an injection of $T \times \mathscr{M}(A)$ into $\mathscr{M}(C(T, A))$. It is shown that if $T$ is realcompact, $A$ is a $Q$ algebra with continuous inversion and either $\mathscr{M}(A)$ is locally equicontinuous or $T$ is discrete, then this injection is a homeomorphism. It is further shown that if the assumption about $T$ is reduced to complete regularity, then $\mathscr{M}\left(C^{*}(T, A)\right)$ is homeomorphic to $(\beta T) \times \mathscr{M}(A)$, and if $A$ is also realcompact, then $\mathscr{M}(C(T, A))$ is homeomorphic to $(\nu T) \times \mathscr{M}(A)$. These results are obtained for topological algebras over the reals, the complexes and certain ultraregular topological fields (including all nonarchimedean valued fields) with no assumptions of local convexity.

1. We assume that the reader is familiar with the properties of $C(T, A)$ and $C^{*}(T, A)$ for $T$ completely regular and $A$ the real or complex numbers, as presented in Gillman and Jerison [4]. For a development of analogous results when $A$ is an ultraregular topological field (an ultraregular space is one whose topology has a base of sets which are both open and closed), the reader is referred to Bachman, Beckenstein, Narici and Warner [1]. In this case, $T$ is also assumed to be ultraregular, the Banaschewski compactification $\left(\beta_{0} T\right)$ is analogous to the Stone-Cech compactification $(\beta T), F$-replete is analogous to realcompact and the $F$-repletion $\left(v_{F} T\right)$ is analogous to the realcompactification $(U T)$. Except where noted, all pairs $(T, A)$ used below are assumed to satisfy either of two sets of conditions: $T$ is completely regular and $A$ is a commutative topological algebra with identity $e$ over the real or complex numbers, or $T$ is ultraregular, $A$ is a commutative topological algebra with identity $e$ over a complete ultraregular topological field $F$, and disjoint $F$-zero sets in $T$ (i.e., inverse images of $\{0\}$ under continuous functions from $T$ into $F$ ) have disjoint closures in $\beta_{0} T$ (which will hold if the field is met- 
rizable). In either case, $F$ denotes the underlying field, and wherever, analogous proofs are applicable they are only written out for the completely regular case. By a $Q$ algebra ( $Q$ ring), we mean inversion is continuous on the open set of units.

Results similar in spirit to $\mathscr{C}(C(T, A)) \cong T \times \mathscr{C}(A)$ have been obtained by various authors. For a topological algebra $X$, let $\operatorname{HOM}(X)=\{M \in \mathscr{C l}(X): M$ is closed $\}$; if $X$ is a $Q$ algebra, $\operatorname{HOM}(X)=$ $\mathscr{C}(X)$. For this paragraph assume that $C(T, A)$ has the compact open topology. Yood showed that if $A$ is a $B^{*}$ algebra and $T$ is compact, then $\operatorname{HOM}(C(T, A)) \cong T \times \operatorname{HOM}(A)$ (cf. [12, Theorem 3.1]); Hausner [5] weakened the condition on $A$ to being a commutative Banach algebra. Using tensor products, Mallios weakened the condition further to $A$ is a locally multipicatively convex (Imc) algebra whose completion is a $Q$ algebra [8, Theorem 5.1]; and (again using tensor products) Dietrich showed that $\operatorname{HOM}(C(T, A)) \cong T \times \operatorname{HOM}(A)$ if $T$ is a completely regular $k$-space and $A$ is a complete locally convex algebra with $\operatorname{HOM}(A)$ locally equicontinuous [3, Theorem 4]. This author showed that $\mathscr{C}(C(T, A)) \cong T \times \mathscr{C}(A)$ if $T$ is realcompact and $A=C(S, F)$ (with the compact open topology) for a locally compact realcompact space $S$ [6, Corollary 2]. In the first three cases, HOM and $\mathscr{C}$ identical, but in the last two HOM $(C(T(A))$ can be a proper subset of $\mathscr{C}(C(T, A))$. Our goal is to generalize the results of Yood, Hausner and Mallios about $\mathscr{C}$ to spaces $T$ which are not compact. No topological structure is imposed on $C(T, A)$, nor are convexity assumptions made about $A$.

2. Fixed ideals. Let $N(f)=\{t \in T: f(t)$ is not invertible $\}$. An ideal $I$ in $C(T, A)$ or $C^{*}(T, A)$ is called free if $\bigcap\{N(f): f \in I\}=\varnothing$ (this is easily seen to be equivalent to Kaplansky's definition of free: for each $t \in T$ there is an $f \in I$ such that $f(t)=e[7$, p. 172]). An ideal is fixed if it is not free. The proofs of the following lemmas are direct.

Lemma 1. Let $m$ be any ideal in $A$ and $t \in T$. Then

( a ) $M_{t, m}=\{f \in C(T, A): f(t) \in m\}$ and $M_{t, m}^{*}=\left\{f \in C^{*}(T, A): f(t) \in m\right\}$ are ideals in $C(T, A)$ and $C^{*}(T, A)$ respectively,

(b) $C(T, A) / M_{t, m}$ and $C^{*}(T, A) / M_{t, m}^{*}$ are algebraically isomorphic to $A / m$,

(c) $M_{t, m}$ and $M_{t, m}^{*}$ are both maximal (of codimension 1) if and only if $m$ is a maximal ideal (resp. of codimension 1 ), and

(d) $M$ is a fixed maximal ideal in $C(T, A)\left(C^{*}(T, A)\right)$ if and only if $M=M_{t, m}$ (resp. $M=M_{t, m}^{*}$ ) for some $t \in T$ and $m$ a maximal ideal in $A$. 
Lemma 2. Let $Y$ be any commutative $F$-algebra with identity, $X$ a subalgebra of $Y$ containing the identity and $m \in \mathscr{M}(Y)$. Then $m \cap X \in \mathscr{C l}(X)$.

Lemma 1 suggests a natural way of identifying points of $T \times \mathscr{C l}(A)$ with ideals in $\mathscr{C}(C(T, A))$ and $\mathscr{C}\left(C^{*}(T, A)\right)$ via the maps $h(t, m)=M_{t, m}$ and $h^{*}(t, m)=M_{t, m}^{*}$. The complete regularity of $T$ insures the injectiveness of $h$ and $h^{*}$, allowing us to consider $T \times \mathscr{C}(A)$ as a subset of both $\mathscr{C}(C(T, A))$ and $\mathscr{C}\left(C^{*}(T, A)\right)$. The set $T \times$ $\mathscr{C}(A)$ then has three topologies under consideration: the product topology, the relative topology from $\mathscr{C}(C(T, A)$ ) (generated by the family $\{\hat{f}: f \in C(T, A)\})$ and the relative topology from $\mathscr{C}\left(C^{*}(T, A)\right)$ (generated by the family $\left\{\hat{f}: f \in C^{*}(T, A)\right\}$ ). The continuity of each $\hat{f}$ with respect to the product topology clearly implies the continuity of $h$ and $h^{*}$. With this in mind, we say that $\mathscr{C}(A)$ is locally equicontinuous if $\mathscr{C l}(A)=\operatorname{HOM}(A)$ and each $m \in \operatorname{HOM}(A)$ has an equicontinuous neighborhood in $\operatorname{HOM}(A)$ (identifying $m \in \mathrm{HOM}(A)$ with the unique continuous multiplicative linear functional of which it is the kernel and $\operatorname{HOM}(A)$ as a subspace of the topological dual of $A$ ).

Lemma 3 (Dietrich [3, p. 208]). Let $\mathscr{L}(A)$ be locally equicontinuous. Then for each $f \in C(T, A), \hat{f}$ is continuous with respect to the product topology; thus $h$ and $h^{*}$ are continuous.

Dietrich also gives an interesting example to show that $h$ need not be continuous if HOM $(A)$ is not locally equicontinuous. The next lemma shows that we can insure the continuity of $h$ and $h^{*}$ by imposing a restriction on $T$ instead of $A$.

Lemma 4. Let $T$ be a discrete topological space. Then for each $f \in C(T, A), \hat{f}$ is continuous with respect to the product topology; thus $h$ and $h^{*}$ are continuous.

Proof. By the definition of the topology on $\mathscr{C}(A), \hat{f}$ is continuous on each slice $\left\{t_{0}\right\} \times \mathscr{C}(A)$. Since these slices form an open partition of $T \times \mathscr{L}(A), \hat{f}$ is continuous on $T \times \mathscr{M}(A)$.

THEOREM 1. Let $T$ be completely regular and $A$ be a commutative topological algebra with identity over the real or complex numbers, or $T$ be ultraregular and $A$ be a commutative topological algebra over a complete ultraregular topological field. Then $h:(t, m) \rightarrow$ $M_{t, m}$ and $h^{*}:(t, m) \rightarrow M_{t, m}^{*}$ are relatively open maps. Therefore if $\mathscr{L}(A)$ is locally equicontinuous or $T$ is discrete, $h$ and $h^{*}$ are homeomorphisms of $T \times \mathscr{M}(A)$ onto subspaces of $\mathscr{C}(C(T, A))$ and 
$\mathscr{L}\left(C^{*}(T, A)\right)$ respectively.

Proof. The product topology is generated by the projections $p_{t}$ and $p_{m}$ onto $T$ and $\mathscr{C}(A)$ respectively. By the complete regularity (ultraregularity in the ultraregular case) of $T$, the topology on $T$ is generated by $C^{*}(T, F)$; by definition the topology on $\mathscr{C}(A)$ is generated by $\{\hat{a}: a \in A\}$. The product topology is then generated by $\left\{g \circ p_{t}: g \in C^{*}(T, F)\right\} \cup\left\{\hat{a} \circ p_{m}: a \in A\right\}$; we claim that each of these is actually of the form $\hat{f}$ for some $\hat{f} \in C^{*}(T, A)$. Consider any $g \in C^{*}(T, F)$ and let $f(t)=g(t) e$; then

$$
\hat{f}(t, m)=g(t) e+m=g(t)=\left(g \circ p_{t}\right)(t, m) .
$$

Next consider any $a \in A$ and let $f(t)=a$; then

$$
\hat{f}(t, m)=a+m=\hat{a}(m)=\left(\hat{a} \circ p_{m}\right)(t, m) .
$$

Thus each function generating the product topology is a function generating both relative topologies, and $h$ and $h^{*}$ are relatively open.

3. The compact case. In the studies of $C(T, F)$ by Gillman and Jerison [4] and Bachman, Beckenstein Narici and Warner [1], the fact that the $F$-zero sets are closed plays a central role. In rings of algebra valued functions, the role of the $F$-zero sets is played by the inverse images of the maximal ideals in $A$ and the set $N$ of all non-invertible elements. It is therefore not surprising to find the assumption that $A$ is a $Q$ algebra appearing in the remaining theorems. That assumption, however, is not necessary: results obtained by this author which were cited above apply when $A=$ $C(S, F)$ (with the compact open topology) for any realcompact and locally compact space $S$. But if $S$ is not compact and $F=C, A$ will not be a $Q$ algebra. For then the functions of compact support are a proper free ideal which must be contained in a maximal ideal; since $S$ is realcompact, a free maximal ideal in $C(S, F)$ cannot be of codimension 1, and therefore is not closed (cf. [9, Prop. 2.9c, p. 13]).

We now show that $h\left(=h^{*}\right.$ in this case) is onto if $T$ is compact and $A$ is a $Q$ algebra; this will then be used in to obtain more general results. We state and prove the theorem only for the real and complex cases. The ultraregular case follows from the following theorem of Kaplansky [7, theorem 24]: if $T$ is a compact ultraregular space and $A$ is a $Q$ ring, then every ideal in $C(T, A)$ is fixed; i.e., $h:(t, m) \rightarrow M_{t, m}$ is onto.

THEOREM 2. Let $T$ be compact and $A$ be a $Q$ algebra over the real 
or complex numbers. Then every proper ideal in $C(T, A)$ is fixed. Thus, $h:(t, m) \rightarrow M_{t, m}$ is onto.

Proof. Suppose $I$ is a free ideal. Then for each $t \in T$ there exists an $f_{t} \in I$ such that $f_{t}(t)=e$. Let $V$ denote the open set of units in $A$, and $U_{t}=f_{t}^{-1}(V)$. Since $T$ is compact, a finite collection $\left\{U_{t_{i}}\right\}$ covers $T$. Let $\left\{h_{i}\right\}$ be a partition of unity subordinate to $\left\{U_{t_{i}}\right\}$, and define $g_{i}(t)=h_{i}(t) f_{t_{i}}(t)^{-1}$ for $t \in U_{t_{i}}$ and $g_{i}(t)=0$ for $t \notin U_{t_{i}}$. Then $g_{i} \in C(T, A)$ and $\sum f_{t_{i}} g_{i} \in I$. Direct computation shows that $\sum f_{t_{i}}(t) g_{i}(t)=e$ for all $t \in T$; therefore $I$ is not a proper ideal.

Corollary 1. If $T$ is compact (compact and ultraregular), $A$ is a $Q$ algebra over the real or complex numbers (resp. over a complete ultraregular topological field) and either $\mathscr{C}(A)$ is locally equicontinuous or $T$ is discrete, then $\mathscr{C}(C(T, A)) \cong T \times \mathscr{C}(A)$.

CoRollary 2(a). If $T$ is completely regular and $A$ is a $Q$ algebra over the real or complex numbers, then the maximal ideals of codimension 1 in $C^{*}(T, A)$ are the ideals $M_{p, m}^{*}=\left\{f \in C^{*}(T, A)\right.$ : $\beta f(p) \in m\}$, where $p \in \beta T$ and $m \in \mathscr{L}(A)$. If $\mathscr{C}(A)$ is also locally equicontinuous, then $\mathscr{L}\left(C^{*}(T, A)\right) \cong(\beta T) \times \mathscr{C}(A)$.

(b) If $T$ is ultraregular and $A$ is a $Q$ algebra over a complete ultraregular topological field, then the maximal ideals of codimension 1 in $C^{*}(T, A)$ are the ideals $M_{p, m}^{*}=\left\{f \in C^{*}(T, A): \beta_{0} f(p) \in m\right\}$, where $p \in \beta_{0} T$ and $m \in \mathscr{C}(A)$. If $\mathscr{C}(A)$ is also locally equicontinuous, then $\mathscr{C l}\left(C^{*}(T, A)\right) \cong\left(\beta_{0} T\right) \times \mathscr{C l}(A)$.

Proof. $f \rightarrow \beta f$ and $f \rightarrow \beta_{0} f$ are isomorphisms from $C^{*}(T, A)$ onto $C(\beta T, A)$ and $C\left(\beta_{0} T, A\right)$ respectively.

Corollary 1 generalizes the theorems of Yood and Hausner. More generally, if $A$ is a locally convex $Q$ algebra, $\mathscr{C}(A)$ is equicontinuous (cf. Warner [11, Theorem 6]), and the preceding results, as well as those which follow, apply. They also apply when $A$ is a locally bounded algebra (cf. Zelasko [13, Chapter 1]). These algebras are not necessarily locally convex.

Theorem 2 shows that if $A$ is a $Q$ algebra and $T$ is compact, then all ideals in $C(T, A)$ are fixed; if $A$ is not a $Q$ algebra in the sense that the set of regular elements of $A$ is not open, it is always possible to find a compact space $T$ such that $C(T, A)$ has free proper ideals. Let $\left\{a_{\alpha}: \alpha \in \Lambda\right\}$ be a net of noninvertible elements of $A$ converging to $e$ and $T=\Lambda \cup\{\infty\}$ the one point compactification of the discrete space 1 . Define $f_{\alpha}(\alpha)=e$ and $f_{\alpha}(t)=0$ for $t \neq \alpha$ and $\alpha \in T$; $f_{\infty}(\infty)=e$ and $f_{\infty}(\alpha)=a_{\alpha}$ for $\alpha \in \Lambda . \quad\left\{f_{\alpha}: \alpha \in T\right\}$ then generates a proper 
free ideal in $C(T, A)$. This ideal is contained in a free maximal ideal, but that ideal may not be of codimension 1 (e.g., if $A=C(S, F)$ with $S$ locally compact, realcompact but not compact).

When $A=F$ and $T$ is realcompact ( $F$-replete in the ultraregular case), $T$ can be recovered from the space of maximal ideals in $C(T, A)$ of codimension 1 (up to a homeomorphism); this is not generally possible for topological algebras $A$, even if $T$ is compact and $A$ is a Banach algebra. Let $S$ be an infinite product of copies of $[0,1]$ with the product topology, $T_{1}=[0,1], T_{2}=\{0\}$ and $A$ the $B^{*}$ algebra $C(S, C)$. By Corollary $1, \mathscr{L}\left(C\left(T_{1}, A\right)\right) \cong T_{1} \times S \cong S$ and $\mathscr{C}\left(\mathrm{C}\left(T_{2}, A\right)\right) \cong$ $T_{2} \times S \cong S$, but $T_{1}$ and $T_{2}$ are clearly not homeomorphic. Furthermore, $T$ cannot be recovered from $C(T, A)$ in any other manner: from a theorem of Yood [12, Theorem 3.1], both $C\left(T_{1}, A\right)$ and $C\left(T_{2}, A\right)$ are isomorphic to $C(S, \mathscr{C})$.

4. The general case. We next examine $\mathscr{l}(C(T, A))$ when $T$ is not compact. As in the case of $C(T, \mathscr{R})$, the realcompactness of $T$ is used to show that all maximal ideals of codimension 1 are fixed and the realcompactification of $T$ is used to "fix" the free maximal ideals of codimension 1. Two preliminary results are needed first (the real and ultraregular cases requiring different proofs): they essentially say that every proper ideal in $C(T, A)$ is "fixed" in $\beta T$.

THEOREM 3. Let $A$ be a $Q$ algebra over the real or complex numbers and $I$ a proper ideal in $C(T, A)$. Then $\bigcap\left\{\mathrm{cl}_{\beta T} N(f): f \in I\right\}$ is not void.

Proof. Suppose that the intersection is empty. Since $\beta T$ is compact, there is a finite set $\left\{f_{i}\right\}$ in $I$ such that $\bigcap\left\{\operatorname{cl}_{\beta T} N\left(f_{i}\right)\right\}=\varnothing$. The complements (in $\beta T$ ) of these sets form a finite open cover of $\beta T$; choose a partition of unity subordinate to that open cover. The restriction to $T$ of these functions is then a partition of unity on $T$ subordinate to the open cover $\left\{T \backslash N\left(f_{i}\right)\right\}$. Denote that partition of unity by $\left\{h_{i}\right\}$ and define $g_{i}(t)=h_{i}(t) f_{i}(t)^{-1}$ for $t$ in $T \backslash N\left(f_{i}\right)$ and $g_{i}(t)=0$ elsewhere. Then $\sum g_{i} f_{i} \in I$ and $\sum g_{i} f_{i}=e$, showing that $I$ is not a proper ideal.

THEOREM 4. Let $A$ be a $Q$ ring, $T$ ultraregular and $I$ a proper ideal in $C(T, A)$. Then $\bigcap\left\{\operatorname{cl}_{\beta_{0} T} N(f): f \in I\right\}$ is not void.

Proof. Proceeding as in the previous proof, we obtain a finite set $\left\{f_{i}\right\}$ in $I$ such that $\beta_{0} T \backslash N\left\{f_{i}\right)$ is an open cover of $\beta_{0} T$. Since $\beta_{0} T$ is ultraregular and compact, this open cover can be refined by a finite clopen partition (a clopen set is one which is both open and 
closed). The characteristic functions of these sets form a partition of unity, and the remainder of the proof is the same as that of Theorem 3.

THeOREM 5(a). Let $T$ be realcompact and $A$ a $Q$ algebra over the real or complex numbers. Then every maximal ideal of codimension 1 is fixed. If $\mathscr{C}(A)$ is locally equicontinuous or $T$ is discrete, then $\mathscr{C}(C(T, A)) \cong T \times \mathscr{C}(A)$.

(b) Let $A$ be a $Q$ algebra over a complete ultraregular topological field and $T$ an ultraragular $F$-replete space in which disjoint $F$-zero sets have disjoint closures in $\beta_{0} T$. Then every maximal ideal of codimension 1 is fixed. If $\mathscr{C}(A)$ is locally equicontinuous or $T$ is discrete, then $\mathscr{C l}(C(T, A)) \cong T \times \mathscr{C l}(A)$.

Proof. Let $M$ be a maximal ideal of codimension 1 in $C(T, A)$. By Theorem 3, there is a $p \in \beta T$ such that $p \in \bigcap\left\{\operatorname{cl}_{\beta T} N(f): f \in M\right\}$. $M \cap C^{*}(T, A)$ is a maximal ideal of codimension 1 in $C^{*}(T, A)$ (Lemma 2); therefore $M \cap C^{*}(T, A)=M_{q, m}^{*}$ with $q \in \beta T$ and $m \in \mathbb{l}(A)$ (Corollary 2). Then

$$
\begin{aligned}
p \in & \bigcap\left\{\operatorname{cl}_{\beta T} N(f): f \in M\right\} \subset \cap\left\{\mathrm{cl}_{\beta T} N(f): f \in M \cap C^{*}(T, A)\right\} \\
& \subset \cap\left\{N(\beta f): f \in M \cap C^{*}(T, A)\right\} \\
= & \{q\}
\end{aligned}
$$

thus $p=q$ and $M \cap C^{*}(T, F)=\left(M \cap C^{*}(T, A)\right) \cap C^{*}(T, F)=m_{p}^{*}=$ $\left\{f \in C^{*}(T, F): \beta f(p)=0\right\} . \quad M \cap C(T, F) \in \mathscr{L} /(C(T, F))$ (Lemma 2), and therefore, by the realcompactness of $T, \quad M \cap C(T, F)=m_{t}=$ $\{f \in C(T, F): f(t)=0\}$, where $t \in T$. Using this, we see that $M \cap C^{*}(T, F)$ is also equal to $m_{t}^{*}$; thus $t=p$. Then $M_{t, m}^{*} \subset M$, whence $M=M_{t, m}$ and $h:(t, m) \rightarrow M_{t, m}$ is onto. Bicontinuity follows from Theorem 1. The proof of part $b$ uses Theorem 4 in lieu of Theorem 3 and is otherwise the same except for notation.

CoRollary 3(a). Let $T$ be completely regular an $A$ a $Q$ algebra over the real or complex numbers which is realcompact. Then the maximal ideals of codimension 1 in $C(T, A)$ are the ideals of the form $M_{p, m}=\{f \in C(T, A): v f(p) \in m\}$, with $p \in v T$ and $m \in \mathbb{C}(A)$. If $\mathscr{L}(A)$ is locally equicontinuous or $T$ is discrete, then $\mathscr{l}(C(T, A)) \cong$ $(u T) \times \cdot \mathscr{C l}(A)$.

(b) Let $A$ be a $Q$ algebra over a complete ultraregular topological field $F, T$ an ultraregular space in which disjoint $F$-zero sets have disjoint closures in $\beta_{0} T$ and $A F$-replete. Then the maximal ideals of codimension 1 in $C(T, A)$ are the ideals of the form $M_{p, m}=$ $\left\{f \in C(T, A): v_{F} f(p) \in m\right\}$, with $p \in v_{F} T$ and $m \in \mathscr{L C}(A)$. If $\mathscr{C l}(A)$ is 
locally equicontinuous or $T$ is discrete, then $\mathscr{C}(C(T, A)) \cong\left(\cup_{F} T\right) \times$ $\mathscr{M}(A)$.

Proof. $f \rightarrow \cup f$ and $f \rightarrow v_{F} f$ are isomorphisms of $C(T, A)$ onto $C(\cup T, A)$ and $C\left(v_{F} T, A\right)$ respectively.

CoROllary 4. If $T$ is a realcompact k-space, $A$ is a locally convex $Q$ algebra over the real or complex numbers and $C(T, A)$ is given the compact open topology, then every maximal ideal of codimension 1 in $C(T, A)$ is closed.

Proof. Use Theorem 5 and Dietrich's theorem [3, Theorem 4].

Note that if $T$ is a $k$-space which is not realcompact, there exist maximal ideals of codimension 1 which are not closed; i.e., $\operatorname{HOM}(C(T, A)) \neq \mathscr{C l}(C(T, A))$. Brooks has examined the relationship between HOM $(X)$ and $\mathscr{C}(X)$ with the hull-kernel topologies for complete lme algebras in [2]. He defines the $X$-realcompactification of $X, v_{X}(\operatorname{HOM}(X))$, in a natural way and proves that $v_{X}(\operatorname{HOM}(X)) \cong$ $\mathscr{C}(X)$ [2, Theorem 1.9]. Call $X$ regular if $\{\hat{x}: x \in X\}$ is a regular family of functions on $\mathscr{C}(X)$ (note that this differs from Brooks terminology in that he only requires it to be a regular family on HOM $(X))$. The proof of Proposition I page 222 in Naimark [10] can be used here to show that the hull kernel topologies on $\mathscr{C}(X)$ and $\mathrm{HOM}(X)$ are the same as the Gelfand topologies used elsewhere in this paper if and only if $X$ is regular. Furthermore, if $X=\mathrm{C}(T, A)$, the imbedding of $C(T, \mathscr{C})$ into $C(T, A)$ via $g(t) \rightarrow g(t) e$ and the complete regularity of $T$ imply the regularity of $C(T, A)$; thus the hullkernel and Gelfand topologies on $\mathscr{C}(C(T, A))$ coincide. We now have a final corollary to Theorem 5 .

CoROllary 5. Let $T$ be realcompact and $A$ be a complete lme $Q$ algebra over the complex numbers, and give $C(T, A)$ any compatible topology which makes it a complete lmc algebra (such as the compact open topology). Then $v_{C(T, A)}(\operatorname{HOM}(C(T, A))) \cong T \times \mathscr{M}(A)$. If $T$ is only assumed to be completely regular, but $A$ is assumed to be realcompact, then $v_{C(T, A)}(\operatorname{HOM}(C(T, A))) \cong(\nu T) \times \mathscr{C l}(A)$.

\section{REFERENCES}

1. G. Bachman, E. Beckenstein, L. Narici and S. Warner, Rings of continuous functions with values in a topological field, Trans. Amer. Math. Soc., 204 (1975), 91-112.

1. R. Brooks, The structure space of a commutative locally m-convex algebra, Pacific J. Math., 25 (1968), 443-454.

3. W. Dietrich, The maximal ideal space of the topological algebra $C(X, E)$, Math. Ann., 183 (1969), 201-212. 
4. L. Gillman and M. Jerison, Rings of Continuous Functions, Van Nostrand, New York, 1960.

5. A. Hausner, Ideals in certain Banach algebras, Proc. Amer. Math. Soc., 8 (1957), 246-249.

6. W. Hery, Maximal ideals in algebras of continuous $C(S)$ valued functions, Atti Accad. Naz. die Lincei, Rend. Cl. Sci. Fis. Mat. Nat., to appear.

7. I. Kaplansky, Topological Rings, Amer. J. Math., 69 (1947), 153-183.

8. A. Mallios, Heredity of tensor products of topological algebras, Math. Ann., 162 (1966), 246-257.

9. E. Michael, Locally multiplicatively-convex topological algebras, Mem. Amer. Math. Soc., 11 (1952).

10. M. Naimark, Normed Rings, Noordhoff, Groningen, the Netherlands, 1964.

11. S. Warner, Polynomial completeness in locally multiplicatively-convex algebras, Duke Math. J., 23 (1956), 1-11.

12. B. Yood, Banach algebras of continuous functions, Amer. J. Math., 73 (1951), $30-42$.

13. W. Zelasko, Metric generalizations of Banach algebras, Rozprawy Math. Warsaw, 47 (1965).

Received June 17, 1975

Polytechnic Institute of New York

Current address: 82-67 88 Lane

Glendale, NY 11227 



\section{PACIFIC JOURNAL OF MATHEMATICS}

\section{EDITORS}

RICHARD ARENS (Managing Editor)

University of California

Los Angeles, California 90024

\section{R. A. Beaumont}

University of Washington

Seattle, Washington 98105
J. DugundjI

Department of Mathematics

University of Southern California

Los Angeles, California 90007

D. Gilbarg and J. Milgram

Stanford University

Stanford, California 94305

\section{ASSOCIATE EDITORS}
E. F. BECKENBACH
B. H. NeumanN
F. WOLF
K. YosHIDA

\section{SUPPORTING INSTITUTIONS}

\author{
UNIVERSITY OF BRITISH COLUMBIA \\ CALIFORNIA INSTITUTE OF TECHNOLOGY \\ UNIVERSITY OF CALIFORNIA \\ MONTANA STATE UNIVERSITY \\ UNIVERSITY OF NEVADA \\ NEW MEXICO STATE UNIVERSITY \\ OREGON STATE UNIVERSITY \\ UNIVERSITY OF OREGON \\ OSAKA UNIVERSITY
}

\author{
UNIVERSITY OF SOUTHERN CALIFORNIA \\ STANFORD UNIVERSITY \\ UNIVERSITY OF HAWAII \\ UNIVERSITY OF TOKYO \\ UNIVERSITY OF UTAH \\ WASHINGTON STATE UNIVERSITY \\ UNIVERSITY OF WASHINGTON \\ AMERICAN MATHEMATICAL SOCIETY
}

The Supporting Institutions listed above contribute to the cost of publication of this Journal, but they are not owners or publishers and have no responsibility for its content or policies.

Mathematical papers intended for publication in the Pacific Journal of Mathematics should be in typed form or offset-reproduced, (not dittoed), double spaced with large margins. Please do not use built up fractions in the text of your manuscript. You may however, use them in the displayed equations. Underline Greek letters in red, German in green, and script in blue. The first paragraph or two must be capable of being used separately as a synopsis of the entire paper. Items of the bibliography should not be cited there unless absolutely necessary, in which case they must be identified by author and Journal, rather than by item number. Manuscripts, in triplicate, may be sent to any one of the editors. Please classify according to the scheme of Math. Reviews, Index to Vol. 39. All other communications should be addressed to the managing editor, or Elaine Barth, University of California, Los Angeles, California, 90024.

The Pacific Journal of Mathematics expects the author's institution to pay page charges, and reserves the right to delay publication for nonpayment of charges in case of financial emergency.

100 reprints are provided free for each article, only if page charges have been substantially paid. Additional copies may be obtained at cost in multiples of 50 .

The Pacific Journal of Mathematics is issued monthly as of January 1966. Regular subscription rate: $\$ 72.00$ a year (6 Vols., 12 issues). Special rate: $\$ 36.00$ a year to individual members of supporting institutions.

Subscriptions, orders for back numbers, and changes of address should be sent to Pacific Journal of Mathematics, 103 Highland Boulevard, Berkeley, California, 94708.

PUBLISHED BY PACIFIC JOURNAL OF MATHEMATICS, A NON-PROFIT CORPORATION

Printed at Kokusai Bunken Insatsusha (International Academic Printing Co., Ltd.), 8-8, 3-chome, Takadanobaba, Shinjuku-ku, Tokyo 160, Japan. 


\section{Pacific Journal of Mathematics \\ Vol. 65, No. $2 \quad$ October, 1976}

Andrew Adler, Weak homomorphisms and invariants: an example .......... 293

Howard Anton and William J. Pervin, Separation axioms and metric-like

functions ............................................. 299

Ron C. Blei, Sidon partitions and p-Sidon sets .................... 307

T. J. Cheatham and J. R. Smith, Regular and semisimple modules ........... 315

Charles Edward Cleaver, Packing spheres in Orlicz spaces .............. 325

Le Baron O. Ferguson and Michael D. Rusk, Korovkin sets for an operator on a

space of continuous functions ............................. 337

Rudolf Fritsch, An approximation theorem for maps into Kan fibrations ....... 347

David Sexton Gilliam, Geometry and the Radon-Nikodym theorem in strict

Mackey convergence spaces .................................

William Hery, Maximal ideals in algebras of topological algebra valued

functions ...................................... 365

Alan Hopenwasser, The radical of a reflexive operator algebra ........... 375

Bruno Kramm, A characterization of Riemann algebras................. 393

Peter K. F. Kuhfittig, Fixed points of locally contractive and nonexpansive

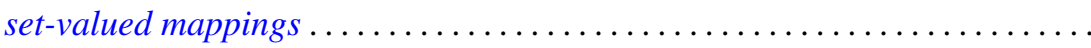

Stephen Allan McGrath, On almost everywhere convergence of Abel means of

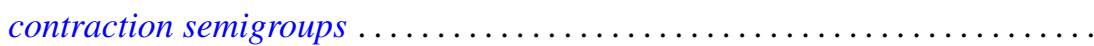

Edward Peter Merkes and Marion Wetzel, A geometric characterization of

indeterminate moment sequences............................ 409

John C. Morgan, II, The absolute Baire property ................... 421

Eli Aaron Passow and John A. Roulier, Negative theorems on generalized convex approximation .................................... 437

Louis Jackson Ratliff, Jr., A theorem on prime divisors of zero and characterizations of unmixed local domains ..............

Ellen Elizabeth Reed, A class of $T_{1}$-compactifications................... 471

Maxwell Alexander Rosenlicht, On Liouville's theory of elementary

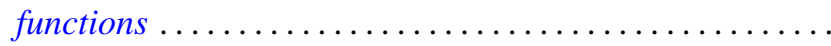

Arthur Argyle Sagle, Power-associative algebras and Riemannian

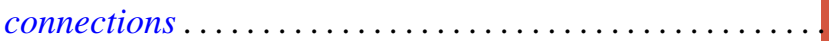

Chester Cornelius Seabury, On extending regular holomorphic maps from Stein manifolds...

Elias Sai Wan Shiu, Commutators and numerical ranges of powers of operators ...................................

Donald Mark Topkis, The structure of sublattices of the product of $n$ lattices ... 525

John Bason Wagoner, Delooping the continuous $K$-theory of a valuation

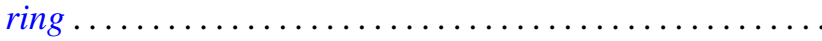

Ronson Joseph Warne, Standard regular semigroups...........

Anthony William Wickstead, The centraliser of $E \otimes_{\lambda} F \ldots$. 